\title{
RESISTENCIA INSTITUCIONAL PARA IMPLEMENTAR LA NUEVA AGENDA URBANA EN PUERTO VALLARTA, MÉXICO
}

\section{INSTITUTIONAL RESISTANCE TO IMPLEMENT THE NEW URBAN AGENDA IN PUERTO VALLARTA, MEXICO}

\author{
BAÑOS FRANCIA, José Alfonso \\ Universidad de Guadalajara (UdG), Centro Universitario de la Costa (CUC) \\ Departamento de Ciencias Exactas, licenciatura en Arquitectura \\ Profesor investigador. \\ Av. Universidad no. 203 delegación Ixtapa, CP. 48280, Puerto Vallarta, Jalisco, México \\ Correo electrónico: jose.banos@academicos.udg.mx, cyberponx@yahoo.com \\ Teléfono: +52 (322) 2262200
}

Palabras clave: territorio turístico, resistencia institucional, Nueva Agenda Urbana.

Key words: tourist territory; institutional resistance; New Urban Agenda

\section{Resumen}

En la participación se exponen las dificultades para implementar la Nueva Agenda Urbana en Puerto Vallarta, destino turístico localizado en la costa del Pacífico mexicano. La narrativa se expone en tres partes. La primera, mostrando los postulados globales sobre desarrollo y ciudad sostenible en torno a los Objetivos de Desarrollo Sostenible (ODS) y la Nueva Agenda Urbana (NAU). En la segunda se presentan los instrumentos de ordenamiento y planeación urbana en Puerto Vallarta. Finalmente, debatiendo sobre las tendencias recientes de crecimiento inmobiliario en el espacio del turismo, donde la autoridad municipal aplica discrecionalmente las normas urbanísticas para favorecer la expansión inmobiliaria vinculada a la actividad turística. Las conclusiones sugieren alentar la participación ciudadana para formular estrategias territoriales que integren los postulados de la Nueva Agenda Urbana en Puerto Vallarta.

La compleja relación entre el territorio y la sociedad está presente en el entorno mundial; en un intento por acceder a procesos de urbanización sostenible, se han presentado esfuerzos globales para implementar el derecho a la ciudad. Un agente activo en estas acciones ha sido la Organización de las Naciones Unidas (ONU), a través de la agencia Hábitat, liderando esfuerzos desde 1976 a partir de la celebración de la Conferencia Mundial sobre los Asentamientos Humanos Hábitat I, celebrada en Vancouver, Canadá. Ello prosiguió 20 años después con Hábitat I/ en Estambul y apenas hace dos años, en Quito se llevó a cabo la reunión Hábitat III. El logro concreto de este último evento fue la publicación de la Nueva Agenda Urbana (NAU), guía de acción acordada por los países de la ONU para el futuro de la vivienda y el desarrollo sostenible en el espacio urbano (ONU, 2016). El documento tiene 17 principios básicos y 175 exhortos generales. De ellos, hay 5 estrategias generales:

1) La necesidad de contar con políticas urbanas nacionales, revalorando el papel del Estado.

2) Actualizar el marco jurídico-legislativo de la urbanización.

3) Disponer de un buen diseño urbano.

4) Encontrar mecanismos de financiamiento para la ciudad.

5) Implementar la NAU a escala local.

Citación: BAÑOS FRANCIA, J. A. Resistencia institucional para implementar la Nueva Agenda Urbana en Puerto Vallarta, México. En: Libro de proceedings, CTV 2018. XII Congreso Internacional Ciudad y Territorio Virtual. "Ciudades y Territorios Inteligentes". UNCuyo, Mendoza, 5-7 septiembre 2018. Barcelona: CPSV, 2018, p. 115-128. 
En México, el gobierno federal incorporó estas estrategias en el marco jurídico expidiendo la Ley General de Asentamientos Humanos, Ordenamiento Territorial y Desarrollo Urbano (LGAHOTyDU) a finales del 2016. La ley contiene principios alineados con el paradigma de una urbanización equitativa como el derecho a la ciudad, cohesión social y participación ciudadana (México, 2016). Sin embargo, estos esfuerzos no son incorporados con el mismo nivel de entusiasmo a nivel municipal, donde los funcionarios muestran poco entusiasmo e interés por acceder a nuevas formas de operar la gestión territorial ${ }^{1}$. Esto se observa en ciudades cuya principal base económica es el turismo, las cuales están sujetas a fuertes presiones inmobiliarias que chocan con la aspiración de contar con mecanismos que equilibren las cargas y beneficios generados por la urbanización. Las conclusiones sugieren alentar la participación ciudadana para formular estrategias territoriales que integren los postulados de la Nueva Agenda Urbana en Puerto Vallarta.

\begin{abstract}
Participation exposes the difficulties to implement the New Urban Agenda in Puerto Vallarta, a tourist destination located on the Mexican Pacific coast. The narrative is exposed in three parts. The first one pf it's, showing the global postulates about development and sustainable city around the Sustainable Development Goals (SDG) and the New Urban Agenda (NAU). In the second, urban planning and planning instruments are presented in Puerto Vallarta. Finally, discussing the recent trends of real estate growth in the tourism area, where the municipal authority discretionally applies the urban regulations to favour real estate expansion linked to tourism. The conclusions suggest encouraging citizen participation to formulate territorial strategies that integrate the postulates of the New Urban Agenda in Puerto Vallarta.
\end{abstract}

The complex relationship between territory and society is present in the global environment; in an attempt to access sustainable urbanization processes, global efforts have been made to implement the right to the city. An active agent in these actions has been the United Nations Organization (UN), through the agency Habitat, leading efforts since 1976 since the celebration of the World Conference on Human Settlements Habitat I, held in Vancouver, Canada. This went on 20 years later with Habitat II in Istanbul and just two years ago, the Habitat III meeting took place in Quito. The concrete achievement of this last event was the publication of the New Urban Agenda (NAU), an action guide agreed by the UN countries for the future of housing and sustainable development in the urban space (UN, 2016). The document has 17 basic principles and 175 general warrants. Of them, there are 5 general strategies:

1) The need to have national urban policies, reassessing the role of the State.

2) Update the legal-legislative framework of urbanization.

3) Have a good urban design.

4) Find financing mechanisms for the city.

5) Implement the NAU at the local level.

In Mexico, the federal government incorporated these strategies into the legal framework issuing the General Law of Human Settlements, Territorial Planning and Urban Development (LGAHOTyDU) at the end of 2016. The law contains principles aligned with the paradigm of equitable urbanization as the right to the city, social cohesion and citizen participation (Mexico,

1 Un ejemplo lo constituye la inasistencia de los funcionarios municipales de Puerto Vallarta a la Conferencia Mundial sobre los Asentamientos Humanos Hábitat I celebrada en Quito en octubre del 2016 y a la resistencia por establecer los principios urbanos contenidos en la LGAHOTyDU. 
2016). However, these efforts are not incorporated with the same level of enthusiasm at the municipal level, where officials show little enthusiasm and interest in accessing new ways of operating territorial management. This is observed in cities whose main economic base is tourism, which are subject to strong real estate pressures that clash with the aspiration of having mechanisms that balance the burdens and benefits generated by urbanization. The conclusions suggest encouraging citizen participation to formulate territorial strategies that integrate the postulates of the New Urban Agenda in Puerto Vallarta.

\section{Introducción}

Puerto Vallarta es un destino turístico localizado en la costa el Pacífico de México que recibió más de cuatro millones de visitantes en 2016; la población se ha duplicado en veinte años y la extensión de la mancha urbana se ha quintuplicado en el mismo periodo.

La complejidad de las dinámicas urbanas a nivel mundial ha alentado el establecimiento de estrategias globales lideradas por la agencia Hábitat de la Organización de las Naciones Unidas (ONU). La más reciente de estas iniciativas es la Nueva Agenda Urbana (NAU), estrategia acordada por los Estados integrantes de la ONU en octubre del 2016.

El documento es una guía de acción para el futuro del desarrollo sostenible y la vivienda; contiene 17 principios básicos y 175 exhortos.

Las estrategias que la NAU propone son cinco: 1) la necesidad de contar con políticas urbanas nacionales, revalorando el papel del Estado; 2) actualizar el marco jurídico-legislativo de la urbanización; 3) disponer de un buen diseño urbano; 4) encontrar mecanismos de financiamiento para las obras prioritarias de la ciudad; 5) implementar la NAU a escala local.

El marco normativo urbano de México se mantuvo sin cambios durante décadas a pesar del crecimiento acelerado de las urbes. Esta debilidad ha sido parcialmente subsanada con la aprobación de la Ley General de Asentamientos Humanos, Ordenamiento Territorial y Desarrollo Urbano (LGAHOTyDU) aprobada en noviembre del 2016, estableciéndose principios relacionados al espíritu de la NAU.

La LGAHOTyDU obliga la actualización de planes y programas de desarrollo urbano en Puerto Vallarta por lo que la autoridad local deberá actualizar 10 planes parciales de desarrollo urbano en sendos distritos.

Adicionalmente, se asiste a una masiva urbanización en espacios turísticos como la llamada Zona Romántica, donde se han modificado discrecionalmente las normas de control contenidas en los planes urbanísticos con el aparente objetivo de favorecer a grupos inmobiliarios bajo un esquema de opacidad y aparente corrupción.

\subsection{Agenda global para la sostenibilidad urbana}

La relación entre territorio y actividades humanas sucede en un entorno donde los sistemas de gobierno son altamente interdependientes. Por ello, en un intento por acceder a procesos de urbanización sostenible, se han formulado diversos esfuerzos institucionales para facilitar los procesos de desarrollo, mencionando algunos de ellos.

Citación: BAÑOS FRANCIA, J. A. Resistencia institucional para implementar la Nueva Agenda Urbana en Puerto Vallarta, México. En: Libro de proceedings, CTV 2018. XII Congreso Internacional Ciudad y Territorio Virtual. "Ciudades y Territorios Inteligentes". UNCuyo, Mendoza, 5-7 septiembre 2018. Barcelona: CPSV, 2018, p. 115-128. 


\subsubsection{La Nueva Agenda Urbana (NAU)}

La Organización de las Naciones Unidas llevó a cabo en 2016 la Cumbre de las Ciudades Hábitat IRen Quito, Ecuador.

La rapidez en el proceso de urbanización a nivel mundial y las implicaciones que tiene sobre los sistemas naturales y humanos sustentaron esta Cumbre, sobre todo porque en el futuro próximo, la mayor cantidad de habitantes vivirán en metrópolis globales. Esto se traduce en una gran presión a la dimensión económica, social y ambiental en las urbes reforzándose los impactos que generan.

Muchos de los esfuerzos de gestión se concretaron en la Nueva Agenda Urbana (NAU), documento que incluye una radiografía de la ciudad contemporánea ofreciendo alternativas para revalorizar el papel estratégico de los asentamientos humanos.

Los trabajos preparatorios de Hábitat III comenzaron en septiembre del 2014 en Nueva York y continuaron en Kenia un año después, concluyendo en julio del 2016 en Surabaya (Indonesia). Como resultado se determinó organizar 7 cumbres temáticas y 4 regionales con una amplia gama de participantes quienes discutieron las prioridades que debían ser incorporadas en la NAU.

Las reuniones temáticas abordaron temas específicos; la primera, denominada Compromiso Ciudadano se desarrolló en Tel-Aviv (Israel) en septiembre del 2015; la segunda, referida a las Áreas Metropolitanas tuvo lugar en Montreal (Canadá); en Cuenca (Ecuador) se llevó a cabo el tercer encuentro con el tema de Ciudades Intermedias; en enero del 2016 se consideró a las Ciudades y Energía Sustentable teniendo como escenario a Abu Dhabi (Emiratos Árabes Unidos); la quinta reunión tuvo como foro a la Ciudad de México bajo el lema Financiamiento del Desarrollo Urbano; el sexto encuentro trató sobre Espacios Públicos en Barcelona y por último, la referida a Asentamientos Informales llevada a cabo en Pretoria (Sudáfrica) en abril del 2016.

Por lo que se refiere a las discusiones regionales, se efectuaron 4; en Yakarta para AsiaPacífico, Abuja (Nigeria) con el tema de África, Praga como sede de Europa y Toluca para América Latina y el Caribe.

Así, la Nueva Agenda Urbana es fundamental para enfrentar temas sensibles como los desafíos en vivienda, infraestructura, provisión de servicios públicos, seguridad, salud, educación, empleo y cuidado ambiental entre otros temas.

Si bien a nivel mundial se reconoce que en los últimos cuarenta años se han logrado avances en el mejoramiento de las condiciones de vida para millones de personas, también persisten múltiples formas de pobreza, desigualdad y degradación ambiental que se traducen en exclusión socio-económica y segregación espacial que afecta el ámbito cotidiano de los habitantes.

Con ello se reafirma el compromiso global para acceder al desarrollo urbano sostenible y para alcanzarlo deben integrarse y coordinarse los diversos actores a escala global, nacional,

${ }^{2}$ Esta conferencia global sobre vivienda y desarrollo urbano se realiza cada 20 años con el objetivo de reforzar el compromiso mundial en la materia. Está precedida por Hábitat l que tuvo lugar en Vancouver (Canadá) en 1976 así como de Hábitat // celebrada en Estambul (Turquía) en 1996. 
regional y local. Este empoderamiento alienta la participación comunitaria en los temas estratégicos para cada contexto, reforzando alianzas y buscando los acuerdos necesarios que le den viabilidad a la tarea de vivir juntos.

En el caso de México, el gobierno federal por medio de la SEDATU (Secretaría de Desarrollo Agrario, Territorial y Urbano) tuvo un papel relevante en la organización de Hábitat III y en la redacción de la NAU. Como resultado, uno de los compromisos adquiridos fue actualizar la ley reglamentaria en materia urbana concretándose por parte del Senado la aprobación de la Ley General de Asentamientos Humanos, Ordenamiento Territorial y Desarrollo Urbano, sustituyendo al instrumento legal formulado hace 40 años.

Por lo que respecta a Puerto Vallarta, el gobierno municipal no mostró interés en incorporar los criterios de sostenibilidad emanados de la NAU. Antes bien, da la impresión que el liderazgo urbano está del lado de los promotores inmobiliarios. En la experiencia local, las élites ajustan las normas urbanísticas para beneficiarse, diluyéndose las fortalezas de un destino turístico que tenía bienes patrimoniales como la imagen urbana tradicional y de baja escala a su favor.

Al adoptar la Nueva Agenda Urbana, se materializa una visión colectiva, así como un compromiso político para promover y alcanzar un desarrollo urbano sostenible remarcando la oportunidad histórica para revalorizar el rol de las ciudades y de los asentamientos humanos como protagonistas del desarrollo sostenible en un mundo en acelerado proceso de urbanización.

\subsection{El territorio y la práctica turística}

El turismo es una de las actividades más importantes y rentables de la economía global (César, Arnaiz y César, 2017); también constituye un potente transformador del territorio debido a su componente espacial, consumiendo suelo, ocupando entornos, modificando el paisaje y transformando los recursos naturales (Baños, 2017b). La dinámica turística genera profundas implicaciones económicas, sociales, culturales y ambientales en las comunidades de acogida (Hiernaux, 2015).

En México, el turismo integra uno de los sectores económicos más importantes por la generación de divisas, creación de empleos y fomento al desarrollo regional (Brenner, 2007). En las ciudades cuya principal vocación es el turismo, se destina una zona para el desarrollo de las actividades recreativas. El origen de los destinos turísticos de playa en México, se debe a dos tipos principales: las ciudades tradicionales de litoral $^{3}$ y los Centros Integralmente Planeados CIP4 (Baños, 2012).

Desde la experiencia mexicana, las ciudades turísticas de litoral se estructuran para la vivencia de los turistas, donde las prácticas se desenvuelven en un espacio cuidadosamente organizado para cumplir con los imaginarios lúdicos y atraer a los visitantes (Méndez, 2012). En esta dinámica, se ha impuesto una frontera invisible que separa a los residentes locales de la costa y los atractivos turísticos, los cuales son disfrutados por los visitantes. En sitios como Puerto

3 El origen de las ciudades tradicionales no se debe primordialmente a la práctica del turismo y que durante su devenir histórico fueron adaptando su estructura socio-espacial para albergar a los visitantes como sucedió en Acapulco, Mazatlán y Puerto Vallarta.

4 Los CIP's fueron desarrollados por el Estado mexicano, a través del Fondo Nacional de Fomento al Turismo (FONATUR), como parte de una estrategia para crear y consolidar diversos mega-proyectos turísticos, a partir de una política articulada y deliberada de mejoramiento territorial y físico; originalmente fueron 5 emprendimientos: Cancún, Los Cabos, Loreto, Ixtapa Zihuatanejo y Huatulco (Dávila, 2015).

Citación: BAÑOS FRANCIA, J. A. Resistencia institucional para implementar la Nueva Agenda Urbana en Puerto Vallarta, México. En: Libro de proceedings, CTV 2018. XII Congreso Internacional Ciudad y Territorio Virtual. "Ciudades y Territorios Inteligentes". UNCuyo, Mendoza, 5-7 septiembre 2018. Barcelona: CPSV, 2018, p. 115-128. 
Vallarta, la estructuración urbana muestra un patrón de división entre el espacio turístico y aquel que no lo es (Baños, 2017a).

Así, el espacio turístico se define como la porción del territorio en la que se desenvuelven actividades recreativas; supone la adaptación del entorno físico para poner en valor los atractivos que originalmente se mantenían en estado prístino o inalterado y que, tras adaptarlos, se incorporan al sistema turístico (Boullón, 1985).

A lo largo de su devenir histórico, las ciudades turísticas de litoral pasan por diferentes etapas que constituyen su ciclo de vida. Una muestra de esta dinámica, se advierte en Puerto Vallarta, que pasó de ser una comunidad agrícola a principios del siglo XX para convertirse en uno de los principales receptores de turistas en México (Cárdenas, 2017).

Esta transformación sucedió mediante la adaptación del medio natural por medio de inversiones públicas y privadas para instalar la infraestructura de soporte que permitiera recibir a los contingentes de turistas.

Pero, el desarrollo turístico mexicano privilegió la cantidad a la calidad. La dialéctica entre turismo masivo o turismo de calidad se inclina hacia la primera modalidad. Los gestores del turismo están más interesados en crecer que en desarrollar el potencial recreativo local. Y para añadirle complejidad, en los lugares de acogida no quedan claros los límites entre el destino turístico y la ciudad, por lo que muchas decisiones de política pública no se toman considerando las necesidades de los residentes locales sino de quienes los visitan (Baños, 2014).

En esta lógica, siempre hay que hablar bien del destino, es políticamente incorrecto señalar los errores o los abusos, hay que conducirse acríticamente para que no se vayan los turistas, ya que sin ellos eléxito turístico quedaría en entredicho. Bajo este discurso, los habitantes locales dejan de asumir su liderazgo y papel protagónico en el proyecto de ciudad que debería incluirlos (Gutiérrez, 2018). Lo usual es que el ciudadano tenga que rendirse, callarse, voltear para otro lado, no importa que personajes y decisiones pongan en entredicho la viabilidad de su ciudad.

Las prácticas extractivas generadas por el turismo y la escasa participación de las instituciones locales para compensar los desajustes son un reflejo de la resistencia a las prácticas sostenibles que dejan pasar la oportunidad de incidir en la promoción y cuidado de los valores comunitarios.

\subsection{Acercamiento a Puerto Vallarta}

Puerto Vallarta es un destino turístico localizado en el litoral del Pacífico mexicano que en el año 2016 recibió más de cuatro millones de visitantes (SETURJAL, 2017). Integra el segundo asentamiento con mayor población en el estado de Jalisco después del Área Metropolitana de Guadalajara (AMG).

De acuerdo a la narrativa histórica, el 12 de diciembre de 1851 Guadalupe Sánchez Torres, arriero y comerciante de sal para las minas de la serranía circunvecina, se estableció en las márgenes del río Cuale, acompañado por familiares y amigos, fundando Las Peñas de Santa María de Guadalupe (Munguía, 1997).

Citación: BAÑOS FRANCIA, J. A. Resistencia institucional para implementar la Nueva Agenda Urbana en Puerto Vallarta, México. En: Libro de proceedings, CTV 2018. XII Congreso Internacional Ciudad y Territorio Virtual. "Ciudades y Territorios Inteligentes". UNCuyo, Mendoza, 5-7 septiembre 2018. Barcelona: CPSV, 2018, p. 115-128. 
El nombre original se conservó hasta el 31 de mayo de 1918, cuando el Congreso de Jalisco lo decretó como municipio modificando su nombre por Puerto Vallarta. El poblado creció, favorecido por la llegada de migrantes de la Sierra Occidental de Jalisco en particular de Mascota, Talpa y San Sebastián del Oeste, teniendo en la agricultura, ganadería y pesca en menor escala como las principales actividades económicas.

La combinación de paisaje serrano y costero, así como la armonía del territorio, favoreció la práctica del turismo durante el siglo XX, generando un polo de desarrollo regional que detonó el crecimiento demográfico con tasas superiores a la media nacional. La población se duplicó en veinte años; en 1990 el número de vallartenses era de 111,457 mientras que en 2010 ascendía a 255,681 habitantes (INEGI, 2010).

La vocación turística de Puerto Vallarta ha supuesto beneficios contribuyendo en la generación de divisas, creación de empleo y fomento del crecimiento regional (Baños, 2012). Sin embargo, por las características y rapidez en el proceso de expansión, se generaron tensiones que se advierten en el incremento de la desigualdad, violencia y la pérdida de identidad y valores culturales.

Actualmente, Puerto Vallarta atraviesa por un periodo de indefinición, caracterizada por una crisis resultante del desgaste en su modelo de turismo masivo. En lo externo, destaca la competencia de otros destinos fuera y dentro de México, el incremento de la violencia vinculada al crimen organizado en ciertas regiones del país, así como el ambiente de estrechez económica mundial (Baños, 2017b).

En lo interno, ha influido un modelo donde los intereses privados se imponen sobre los colectivos, malas decisiones de política pública, un sistema turístico depredador de la economía local e incapacidad para innovar el producto recreativo.

\subsubsection{Instrumentos de planeación urbana en Puerto Vallarta}

El primer instrumento de ordenamiento urbano en Puerto Vallarta fue el Plan General Urbano aprobado en 1975 y elaborado por la Junta General de Planeación y Urbanización de Jalisco (JGPU). En el documento se reconocía la importancia de la actividad turística y de los retos por enfrentar, como el acelerado crecimiento demográfico, la expansión urbana y la presencia de una importante población flotante; de continuar el ritmo de crecimiento, se asumirían graves problemas naturales y urbanos (JGPUEJ, 1975).

Posteriormente, en 1997 fue aprobado el Plan de Desarrollo Urbano (PDU) para establecer medidas de acción urbanística con el fin de regular y controlar las actividades de conservación, mejoramiento y crecimiento urbano. Este documento prevaleció por más de quince años y contribuyó en el ordenamiento del territorio vallartense, sin embargo, sus metas fueron rebasadas por la fuerte expansión urbana dificultando la promoción de un desarrollo territorial (Baños, 2015).

En los años 2003, 2006 y 2009 se llevaron a cabo tres intentos institucionales para actualizar el PDU pero que todos los procesos fueron bloqueados por la falta de consensos entre actores locales. Esto derivó en la judicialización de la planeación urbana, particularmente con el Plan

Citación: BAÑOS FRANCIA, J. A. Resistencia institucional para implementar la Nueva Agenda Urbana en Puerto Vallarta, México. En: Libro de proceedings, CTV 2018. XII Congreso Internacional Ciudad y Territorio Virtual. "Ciudades y Territorios Inteligentes". UNCuyo, Mendoza, 5-7 septiembre 2018. Barcelona: CPSV, 2018, p. 115-128. 
de Desarrollo Urbano aprobado en $2006^{5}$. El impedimento se mantuvo vigente hasta diciembre del 2011 cuando se dictó sentencia definitiva declarándose el sobreseimiento del juicio, oficializándose la validez jurídica del Plan y ordenando su cumplimiento y observancia en el centro de población. De esta manera, el PDU en cuestión rige actualmente la zonificación primaria en el centro de población de Puerto Vallarta.

Con el ánimo de actualizar la gestión urbana, el gobierno municipal aprobó en 2011 dividir la zona urbana en 10 distritos dentro del Límite de Centro de Población (LCP). ${ }^{6}$ La división por distritos impulsó la formulación de Planes Parciales de Desarrollo Urbano (PPDU) ${ }^{7}$ para detallar los instrumentos de ordenamiento y adecuarlo a las exigencias de cada zona. Los esfuerzos se tradujeron en la aprobación de diez PPDU ${ }^{8}$ en 4 años, lo cual constituyó un avance significativo en la práctica urbanística de Puerto Vallarta.

Desafortunadamente, pese a contar con los PPDU aprobados, en la práctica no se han respetado los postulados de la planeación urbana dando la impresión de que la marea especulativa del sector inmobiliario se impone doblegando a la autoridad y a la normatividad, en particular en el espacio turístico. Un recurso muy socorrido ha sido modificar discrecionalmente las reglas urbanísticas alegando que los planes no corresponden con la realidad del contexto ${ }^{9}$. Para hacer frente a estos abusos, diversas asociaciones de vecinos, en particular en la franja turística ${ }^{10}$, han intentado colaborar en la gestión urbanística.

Otra debilidad de la planeación urbana en Puerto Vallarta es que los instrumentos se formulan con un enfoque positivista sin considerar la complejidad del territorio, basándose en la zonificación como la herramienta para conducir el crecimiento; si bien este método de ordenamiento se aplicó en el auge del crecimiento urbano surgido tras la Revolución Industrial, en los tiempos actuales muestra su incapacidad para absorber y regular las profundas y veloces transformaciones en el espacio urbano (García, 2016).

Y una vez que se cuenta con planes urbanísticos pertinentes, hay que gestionar adecuadamente su implementación, aspecto que se ha evitado por una mezcla ineficiente de especulación inmobiliaria, corrupción gubernamental, intereses de poder y escasa participación

5 Durante la administración municipal 2004-2006, la Asociación de Hoteles y Moteles, así como otros organismos empresariales interpusieron una demanda de nulidad al Plan de Desarrollo Urbano aprobado ante el Tribunal Administrativo de Jalisco (TAE), ordenándose la suspensión.

6 El Límite de Centro de Población (LCP) comprende las áreas dentro de las que se aplican los instrumentos de planeación urbana. En el caso de Puerto Vallarta, incluye una superficie de 10,581-50-56.18 hectáreas. Para delimitar los distritos, se adoptó una metodología que considera las características del medio natural, urbano y socio-económico, advirtiéndose algunos desajustes como la distribución poblacional desequilibrada en ciertas demarcaciones.

7 De acuerdo al Código Urbano para el Estado de Jalisco, el Plan Parcial de Desarrollo Urbano (PPDU) tiene como objetivo precisar la zonificación y normas de utilización de predios regulando detalladamente la urbanización en el área de aplicación. Con ello, se determina la zonificación a nivel secundario.

8 Los Planes Parciales de Desarrollo Urbano aprobados fueron 1a (Río Ameca), 2 (Ixtapa), 3 (Mojoneras), 4 (El Pitillal), 5a (Marina Vallarta), 5b (Estero El Salado), 6 (Zona Hotelera Norte), 9 (Amapas-Conchas Chinas), 10 (Zona Sur) y Arroyo El Quelitán, éste último fuera del Límite de Centro de Población.

9 Basados en una interpretación del artículo 35 del Reglamento Municipal de Zonificación, promotores de desarrollos pueden solicitar a la autoridad municipal la modificación de las normas de control de la edificación contenida en los planes urbanos mediante la presentación de un Análisis Técnico Justificativo (ATJ). Esta facultad discrecional ha facilitado la alteración de los tejidos urbanos, sobre todo en la franja turística, que es el espacio de mayor atracción para la inversión inmobiliaria.

10 Las asociaciones de vecinos más activas en materia urbana son Amapas y Conchas Chinas. Ambas están integradas mayoritariamente por extranjeros que se han involucrado en la temática urbana motivados por la conservación de la plusvalía de su inversión, en la dinámica conocida como NIMBY (Not in my Backyard). Su participación incluye mejoras físicas a las colonias, así como instrumentos de gestión urbana como la declaratoria de Polígono de Desarrollo Controlado (PDC).

Citación: BAÑOS FRANCIA, J. A. Resistencia institucional para implementar la Nueva Agenda Urbana en Puerto Vallarta, México. En: Libro de proceedings, CTV 2018. XII Congreso Internacional Ciudad y Territorio Virtual. "Ciudades y Territorios Inteligentes". UNCuyo, Mendoza, 5-7 septiembre 2018. Barcelona: CPSV, 2018, p. 115-128. 
ciudadana. Así, es deseable promover la transparencia en la formulación e implementación del ordenamiento urbano, donde el gobierno local encabece la actualización de los planes y la rendición de cuentas, apoyado en organizaciones y ciudadanos. La inoperancia de un modelo urbano marcado por la especulación individual puede ser superada por otras modalidades de actuación colectiva para acceder a un desarrollo sustentable del territorio en Puerto Vallarta.

\subsubsection{Principios para una urbanización equitativa en ciudades turísticas de litoral}

Hay tres principios sobre los que podrían apoyarse los procesos de urbanización en las ciudades turísticas de litoral en México.

El primero es el valor social del suelo consignado en el artículo 27 de la Constitución Política de los Estados Unidos Mexicanos; desafortunadamente el mundo de consumo alteró este valor transformándolo en una moneda de cambio, en un producto mercantil. La relación entre territorio y sociedad comenzó a desajustarse cuando a la tierra se le puso un precio en vez de conservar su valor.

El segundo principio es que la urbanización genera valor y ella debe ser re-distribuida entre todos los habitantes no solamente entre algunos beneficiarios, máxime si se trata de inversionistas inmobiliarios. Si en los destinos nacionales se hubiera capturado un porcentaje de la plusvalía generada por la edificación de la planta turística, se podrían haber obtenido recursos para financiar obras de infraestructura, equipamiento y espacio público en los entornos municipales.

La responsabilidad gubernamental para promover equidad social y generar iniciativas redistributivas no ha sido una prioridad en la agenda pública, a pesar de que se cuentan con muchos ejemplos de buenas prácticas establecidas por gobiernos locales en el mundo en desarrollo en particular en América Latina (McGuirk, 2015).

El tercer principio es contar con políticas urbanas locales que se traduzcan en instrumentos eficientes de planeación urbana para otorgar racionalidad al territorio (Azuela, 2013). En la práctica mexicana, se observa la incorporación de los principios urbanos acordados por organismos globales como ONU Hábitat y que están redactados en la Nueva Agenda Urbana o los Objetivos de Desarrollo Sostenible, los cuales son promovidos a nivel federal pero cuya intención se diluye al implementarlos en los municipios.

Por lo que respecta a Puerto Vallarta, la ciudad ha estado sometida a profundos procesos de trasformación desde la década de 1970. A veces la dinámica se ha acelerado o disminuido, pero nunca ha dejado de experimentar poderosos cambios (Baños, 2010).

\subsubsection{Tendencias de urbanización recientes en Puerto Vallarta}

A partir del año 2010, los procesos de transformación urbana en la franja turística se han acelerado, en particular en la colonia Emiliano Zapata, en la llamada Zona Romántica y en otros sitios como Marina Vallarta, Amapas, Altavista y Conchas Chinas.

Al caminar por las calles de la Zona Romántica se advierte el contraste cada vez más impactante entre el paisaje natural y construido. La montaña cubierta de vegetación cede su lugar, paulatinamente a nuevas torres departamentales cuyos residentes, de acuerdo a

Citación: BAÑOS FRANCIA, J. A. Resistencia institucional para implementar la Nueva Agenda Urbana en Puerto Vallarta, México. En: Libro de proceedings, CTV 2018. XII Congreso Internacional Ciudad y Territorio Virtual. "Ciudades y Territorios Inteligentes". UNCuyo, Mendoza, 5-7 septiembre 2018. Barcelona: CPSV, 2018, p. 115-128. 
investigaciones empíricas, suelen ser temporales al visitar el destino solamente por temporadas (Baños y Huízar, 2016).

Sólo en la Zona Romántica se han edificado 20 desarrollos en los últimos 7 años que suman más de 11 hectáreas construidas; ésta masiva urbanización representa el $56.54 \%$ de todo lo que se construyó en su historia.

Tabla 1. Desarrollos recientes en Zona Romántica

\begin{tabular}{|c|c|c|c|c|}
\hline Desarrollo & Estatus & $\begin{array}{c}\text { Superficie } \\
\text { construida } \mathrm{m}^{2}\end{array}$ & $\begin{array}{c}\text { Unidades } \\
\text { privativas }\end{array}$ & $\begin{array}{c}\text { Unidades privativas } \\
\text { normativa }^{*}\end{array}$ \\
\hline Pacífica & Concluido & $3,846.00$ & 36 & 5 \\
\hline The Park & Concluido & $7,982.10$ & 54 & 10 \\
\hline Zenith & Concluido & $9,672.00$ & 44 & 10 \\
\hline V399 & Concluido & $5,096.00$ & 55 & 6 \\
\hline Pinnacle 1 & Concluido & $8,607.20$ & 48 & 9 \\
\hline Pinnacle 2 & Concluido & $7,586.24$ & 57 & 8 \\
\hline V177 & Concluido & $6,722.80$ & 48 & 9 \\
\hline D'Terrace & Concluido & $8,006.60$ & 43 & 6 \\
\hline Rincón de Almas & En proceso & $3,511.20$ & 38 & 5 \\
\hline Pier 57 & En proceso & $9,821.00$ & 69 & 4 \\
\hline Oceana & En proceso & $5,205.85$ & 42 & 6 \\
\hline Nayri & En proceso & $6,939.72$ & 54 & 9 \\
\hline Loft 268 & En proceso & $9,440.99$ & 64 & 10 \\
\hline Aguacate & Concluido & $1,203.42$ & 11 & 4 \\
\hline Isla Cuale & En proceso & $3,619.00$ & 30 & 10 \\
\hline Pavillon & En proceso & $8,209.59$ & 62 & 10 \\
\hline V Estrella & En proceso & $6,462.58$ & 23 & 21 \\
\hline Basilio Badillo 418 & En proceso & 513.02 & 7 & 3 \\
\hline Lázaro Cárdenas 378 & En proceso & $1,037.92$ & 11 & 4 \\
\hline 105o Sail View & En proceso & Sin datos & 53 & --- \\
\hline Total & & $113,483.23$ & 847 & 179 \\
\hline
\end{tabular}

* Considerando el Plan Parcial de Plan Parcial de Desarrollo Urbano "Los Muertos" aprobado en 1999 (vigente) y Plan de Desarrollo Urbano de Centro de Población 2006 (vigente).

Fuente: Elaboración propia, basada en investigación empírica, así como licencias de construcción de los desarrollos señalados proporcionados por la Dirección de Planeación Urbana y Ecología del H. Ayuntamiento de Puerto Vallarta.

La intensa transformación ha traído aspectos positivos como la atracción de inversión, reforzamiento de la centralidad y activación de actividades económicas. Pero también supone retos como la transformación del paisaje urbano, la sobre-densificación y la generación de una economía de enclave que confina a residentes y visitantes.

¿Qué ha disparado la potente transformación reciente...? Uno de los principales detonadores es la facilidad para rentar viviendas turísticas en sitios electrónicos de hospedaje como AirBnB, Coach Surfing o Booking. Ahora los propietarios pueden ofertar sus propiedades por internet y obtener ingresos extras que permitan financiar la adquisición de sus unidades ${ }^{11}$. Ello, es la causa que aceita el tsunami urbanizador reciente.

11 De acuerdo a información empírica obtenida mediante entrevistas a desarrolladores inmobiliarios y propietarios de departamentos, el arrendamiento de hospedaje en plataformas digitales genera ingresos anuales promedio de $\$ 25$ mil dólares estadounidenses; así, con estos ingresos, en una década es posible pagar el costo de las unidades. 


\subsubsection{Resistencia institucional para implementar la Nueva Agenda Urbana}

Los principios del urbanismo sostenible expresados en la Nueva Agenda Urbana no son implementados en Puerto Vallarta por una resistencia institucional, aunque en el discurso oficial se aspira llegar a una ciudad verde y justa (Ayuntamiento de Puerto Vallarta, 2015).

La resistencia se da entre actores gubernamentales, empresariales, gremiales (profesionales vinculados al sector de la construcción) y sociales (juntas de vecinos). El poder público parece olvidar su obligación de alentar la prosperidad colectiva para favorecer a ciertos grupos empresariales, quienes, a su vez, recompensan generosamente las gestiones de los responsables. Da la impresión de que el gobierno municipal es un agente inmobiliario más, en un facilitador de negocios.

Esto se refleja en una holgada discrecionalidad en la toma de decisión urbana, modificándose de manera sostenida las normas de control de los planes urbanos, a partir de interpretaciones flexibles sobre políticas nacionales (como la densificación) o locales (generación de empleo mediante el apoyo a los inversionistas turísticos). La planeación urbana no tiene sentido si no se respetan las normas del juego contenida en dichos ordenamientos.

De los 20 desarrollos inmobiliarios mencionados en la Zona Romántica, en todos los casos se presentaron Análisis Técnicos Justificativos (ATJ) para alterar al alza, los coeficientes constructivos. Esto significa que, de acuerdo a las normas urbanísticas en la Zona Romántica hubiera sido posible construir solamente 179 unidades privativas (departamentos) pero en la realidad se autorizaron 847 departamentos (Tabla 1), es decir, 668 más de lo estipulado, equivalente a $373 \%$ más $^{12}$.

Con ello, se ha dejado pasar la oportunidades para aprovechar la valorización generada por las construcciones recientes para capturar la plusvalía y transferirla mediante instrumentos compensatorios a aquellas zonas de la ciudad que requieran de inversión en infraestructura básica, espacio público o movilidad. En Puerto Vallarta, el organismo operador del agua señala que requiere 60 millones de pesos urgentes para rehabilitar tramos de la red de drenaje solamente en la Zona Romántica. Paradójicamente, el gobierno municipal aplica programas asistencialistas de mejora a la vivienda que se aplican en los barrios marginarles basados en la dotación de materiales de construcción, pero cuya efectividad es cuestionable al no resolver de raíz el problema de la desigualdad y la pobreza.

Además, se carece de políticas urbanas consensadas y construidas en colectivo para fijas estrategias que consideren al territorio no solamente como la base física para el desarrollo de las actividades básicas sino como un sistema de relación básico para promover la prosperidad colectiva. La falta de discusión de los asuntos públicos impide la construcción de acuerdos que le den viabilidad al futuro, antes bien, hay una tendencia institucional para acallar las voces críticas recurriendo a la censura, descalificación y la amenaza. Cuando no hay proyecto de ciudad o los objetivos no quedan claros, la gestión urbana es poco eficiente y se diluye.

Finalmente, no se observa la adopción de mecanismos de integración residencial, antes bien, se incrementa la gentrificación, segregación y exclusión en la vivencia del espacio urbano vallartense (Baños, 2017b) particularmente en la franja turística, construida con fragmentos

12 El costo promedio de cada unidad (departamento) es de $\$ 250$ mil dólares estadounidenses, equivale a unos $\$ 167$ millones de dólares, es decir, unos 3 mil millones de pesos mexicanos. Es muy difícil, en estos tiempos de neoliberalismo económico, resistir los cañonazos de la industria inmobiliaria. 
aislados donde aparece el resentimiento entre privilegiados y excluidos, entre turistas y trabajadores, en particular quienes realizan trabajos poco remunerados en el sector turístico (Gutiérrez, 2018).

Y el Estado, lejos de buscar mecanismos compensatorios para disminuir los picos de desigualdad, fortalece al poderoso otorgándole derechos que van en contra de la solidaridad y prosperidad colectiva. Hoy la ciudad y en particular, la ciudad turística divide en lugar de unir, las calles son trincheras y no espacios para el encuentro y el intercambio.

\section{Apuntes finales}

La expectativa de construir una sociedad más humana y justa, que nos acerque a otro mundo posible, pertenece a millones de personas (Jacobs, 1961). Personas que no tienen voz, ni poder, ni relaciones, ni figuran en los circuitos del poder. Es posible modificar el estado de las cosas y proponer nuevas maneras de incidir en el territorio buscando equilibrar las cargas y beneficios (Baraona y Reyes, 2017).

La transformación puede ser rápida, pero implica modificar las prácticas dominantes basadas en la acumulación económica. Mientras no se reforme este esquema, seguirán los abusos territoriales y la modificación discrecional de las normas del juego, sin importar el origen político de la autoridad en turno. Aminorar la mentalidad extractiva arraigada por siglos, será un reto interesante que veremos resistir en las próximas décadas.

Da la impresión que los rieles de la locomotora del mundo están aceitados para que siga imperando la acumulación del capital en manos privadas. Parece que lejos de armonizar la velocidad de la nave, hay que acelerarla para mantener el ritmo de crecimiento a costa de las externalidades generadas (Secchi, 2013). La pregunta es si podremos sostener el ritmo sin costos añadidos, sin daños colaterales, en un ambiente poco propicio a la discusión sobre la velocidad apropiada para el desarrollo. En caso de que se tuviera la fórmula para afinar el ritmo deseado, hay que contar con mecanismos pertinentes para evaluar la evolución de esta realidad cambiante y compleja.

El modelo económico actual hace corto circuito con las aspiraciones de la sostenibilidad en sus tres dimensiones (social, ambiental y económica). Hay una profunda contradicción entre crecer y conservar. Un camino alternativo es ralentizar la expansión, apostar por el de-crecimiento. Desde la economía se dirá que hay que crecer hasta el infinito y que la pobreza disminuirá hasta que se alcancen niveles de consumo similares a los países hegemónicos. Poco se insiste en los satisfactores ambientales y sociales, que son necesarios para completar la tríada de la sostenibilidad.

Otra realidad urbana es posible, pero requiere del concurso y esfuerzo de la comunidad. En la esfera urbana, las luchas siempre serán buenas, aunque parezcan perdidas. El cambio de gran calado vendrá de la participación ciudadana, activa, informada, cuestionante, difícilmente vendrá de las élites, para quienes el estado de las cosas es conveniente. Las ciudades turísticas de litoral en México deben contar con instrumentos de ordenamiento territorial y mecanismos de gestión urbana con criterios de sustentabilidad. Y en Puerto Vallarta también se puede transitar por caminos alternativos para promover la convivencia, fraternidad y prosperidad. 


\section{Bibliografía}

AyUntamiento De PUERTO VAlLARTA. Plan de Desarrollo Municipal. Puerto Vallarta, Ayuntamiento de Puerto Vallarta, 2015.

BAÑos, A. Segregación residencial en el espacio turístico de Puerto Vallarta. Guadalajara: Universidad de Guadalajara, 2017a.

BAÑoS, A. Políticas para el territorio en Puerto Vallarta, Jalisco. La implementación incompleta del ordenamiento en el espacio del turismo. En: Arellano, A. (coord.) Políticas públicas y territorio. Diseño e implementación de programas gubernamentales en Jalisco, 2017b. Guadalajara: Universidad de Guadalajara y Colegio de Jalisco, pp. 153-182.

BAÑOS, A. El declive del Centro Histórico en Puerto Vallarta, México. Una aproximación para la rehabilitación. En: Contexto, XI (15): 69-81. 2017c. [En línea] Disponible en: https://www.redalyc.org/html/3536/353652711006/

BAÑOS, A. Planeación y políticas urbanas en Puerto Vallarta, En: Estudios Jaliscienses, 101: 30-41. 2015.

BAÑoS, A. Turismo, identidad y espacio público en Puerto Vallarta, México. Apuntes sobre tres intervenciones recientes. En: PASOS. Revista de Turismo y Patrimonio Cultural, 12, (2): $491-$ 498. 2014. \{En Línea] Disponible en: https://www.redalyc.org/articulo.oa?id=88130205001

BAÑOS, A. La modernidad persistente y la escenografía de la tradición en la arquitectura de Puerto Vallarta. En: Méndez, E.; González, D.; Olivares, A. y PÉREZ, M. T. (coords.) Arquitectura moderna desde la calle: un recorrido de ciudades, 2013. Guadalajara: Universidad de Guadalajara, pp. 329-338.

BAÑOS, A. Ocupación del territorio litoral en ciudades turísticas de México. En: Bitácora Urbano Territorial, 20 (1): 41-52. 2012. [En línea] DOI: http://dx.doi.org/10.15446/bitacora Disponible en: https://revistas.unal.edu.co/index.php/bitacora/article/view/24860

BAÑos, A. Arquitectura y urbanismo en Puerto Vallarta. Una mirada a la construcción de una ciudad turística de litoral. Puerto Vallarta: Instituto Tecnológico Superior de Puerto Vallarta. 2010.

BAÑos, A. y HUÍZAR, M. de los Á. Territorios turísticos de la diversidad. En: Ciudades, 112 (Turismo urbano y su papel en la planeación de las ciudades): 39-47. 2016.

BARAONA, E. y REYES, C. El futuro será obsoleto. En: Futuros. México: Arquine, 37-42. 2017

BOULLÓN, R. C. Planificación del espacio turístico. México: Trillas. 1985.

CARRERO DE ROA, M. Fundamentos de urbanismo. Una perspectiva sostenible. España: Ojo x Hoja. 2010.

CÁRDENAS, É. Las políticas turísticas en México y su aplicación en la zona metropolitana de Puerto Vallarta. En: Arellano, Alberto (coord.) Políticas públicas y territorio. Diseño e implementación de programas gubernamentales en Jalisco. Guadalajara: Universidad de Guadalajara y Colegio de Jalisco, pp. 123-152. 2017.

Citación: BAÑOS FRANCIA, J. A. Resistencia institucional para implementar la Nueva Agenda Urbana en Puerto Vallarta, México. En: Libro de proceedings, CTV 2018. XII Congreso Internacional Ciudad y Territorio Virtual. "Ciudades y Territorios Inteligentes". UNCuyo, Mendoza, 5-7 septiembre 2018. Barcelona: CPSV, 2018, p. 115-128. 
CÉSAR, A.; ARNAIZ, S. M. y CÉSAR, F. (2017). Capitalismo, sociedad y turismo. México: Universidad de Guadalajara. 2017.

DÁVILA, A. La otra Marcha al Mar. Territorios costeros colonizados. Interpretando los centros turísticos de playa mexicanos de FONATUR. Tesis de Maestría. Barcelona: Universidad Politécnica de Cataluña. 2015.

GARCÍA CANCLINI, N. ¿El soborno es el nuevo contrato social? En: Habitar la ciudad, México: Arquine, 77-84. 2016.

GARCÍA VÁZQUEZ, Carlos. Teorías e historia de la ciudad contemporánea. España, Gustavo Gili. 2016.

GUTIÉRREZ, N. Actitudes del residente ante el turismo sostenible. Adaptación y validación de una escala de medida. Tesis de Maestría. México: Centro Universitario de la Costa, Universidad de Guadalajara. 2018.

HERCE, M. El negocio del territorio. Evolución y perspectivas de la ciudad moderna. España: Alianza. 2013.

HIERNAUX, D. Fantasías, sueños e imaginarios del turismo contemporáneo. En: Hiernaux, Daniel (coord.). Turismo, sociedad y territorio: una lectura crítica. México; Universidad Autónoma de Querétaro, 65-98. 2015.

JACOBS, J. The death and life of Great American Cities. España: Swing libros. 1961.

JGPUEJ [Junta General de Planeación y Urbanización del Estado de Jalisco]. Plan General Urbano de Puerto Vallarta. Guadalajara: Gobierno de Jalisco. 1975

MÉNDEZ, E. Imaginario de ciudad turística: una propuesta de abordaje, en Enríquez, Jesús y Méndez, Eloy (coords.) De itinerarios, paisajes e imaginarios. Miradas y acercamientos al estudio del turismo. Hermosillo: Universidad de Sonora, pp. 11-44. 2012.

MÉXICO. Ley General de Asentamientos Humanos, Ordenamiento Territorial y Desarrollo Urbano. México, Gobierno Federal. 2016.

MONTANER, J. M. La condición contemporánea de la arquitectura. Barcelona, G. Gili. 2015.

MONTANER, J. M. y MUXÍ, Z. Arquitectura y política. Ensayos para mundos alternativos. Barcelona, Gustavo Gili. 2011.

MUNGUíA, C. Panorama histórico de Puerto Vallarta y de la Bahía de Banderas, Puerto Vallarta. México: Secretaría de Cultura del Gobierno de Jalisco y H. Ayuntamiento de Puerto Vallarta. 1997.

ONU-HÁBITAT. Nueva Agenda Urbana. Nairobi, ONU publicaciones. 2016.

SETURJAL. Secretaría de Turismo de Jalisco. Resultados de ocupación en Puerto Vallarta. Documento interno. 2017

VALENZUELA, A. Urbanistas visionarios. La planeación de la Ciudad de México en la primera mitad del siglo XX. México: Porrúa. 2014. 